\title{
COMMENTS SECTION
}

\section{A word from the editor about this new MEPS feature}

MEPS strives to further marine sciences by making judicious use of Comments Sections (CSs): series of invited, brief, coordinated and pre-edited comments on a selected 'cutting edge' topic. CSs should clearly formulate the information offered, and-where appropiate-outline alternatives and perspectives; they must not become personal or unnecessarily harsh. CSs can be-sometimes more so than individual papersinstrumental in focussing attention, triggering opin- ions and stimulating ideas, discussions and activities in special research fields. CSs, normally initiated and organized by a Senior Advisor of MEPS, must be carefully planned and not excessive in number (e.g. not more than 10 per year). Hence, it is necessary to contact me before starting to work on a CS. While a CS is not subject to normal peer review procedures, it may be desirable to invite opinions from outside experts.

Otto Kinne, Editor MEPS

\section{Predator-prey interactions in the sea: commentaries on the role of turbulence}

\section{Howard I. Browman}

Department of Fisheries and Oceans Canada, Maurice-Lamontagne Institute, Ocean Productivity Division, PO Box 1000, Mont-Joli, Quebec, Canada G5H 3Z4

E-mal: h_browman@qc.dfo.ca

Recent work on turbulence is at the heart of refined interpretations of particle (and predator-prey) encounter rates in water columns. As evidenced by the rapidly growing number of publications devoted to this subject, turbulence has received significant attention from researchers and is currently a major issue in marine ecology.

Much of the work on turbulence is based upon a paper published by Brian Rothschild and Thomas Osborn in 1988. Several other authors have modified and/or extended the relationships put forward in Rothschild \& Osborn (1988) (reviewed by Dower et al. in press). However, all evaluations of the effects of turbulence on particle encounter rates are based upon similar fundamental assumptions.

From the physicist's end of things, some of the key issues are: (1) The choice of $d$, the distance between 2 points (particles) for which a velocity difference $(w)$ due to fluid motion is to be calculated. The value chosen for $d$ is central to evaluations of turbulenceinduced particle encounter rate. In a functional sense, and for the particular group(s) of organisms being considered, what is an appropriate choice for $d$ ? Is it the mean separation distance between particles (predator and prey)? The maximum distance at which the predator can perceive prey (i.e. the reaction distance)? Does it make any difference which one is chosen? (2) The assumption that the relationship proposed for $w$ in Rothschild \& Osborn (1988) holds at values of $d$ below the Kolmogorov scale, or at least at those scales relevant to the interactions of planktonic organisms. (3) The assertion that turbulence can be accurately measured in the sea and that realistic turbulent energy spectra can be generated (and measured) in the laboratory.

From the behaviourist's end of things, some of the fundamental issues are: (1) The choice of geometry and volume for the predator's prey field. (2) The interpretation and modelling of the predator's prey search pattern. (3) The determination of which component(s) of the predation cycle is affected by turbulence, and how.

Few of these issues/assumptions have been subjected to rigorous scrutiny. Further, analytical models of the effects of turbulence on predator-prey encounter rate are rarely presented along with some form of validation or in-depth analysis of their sensitivity to relaxation of their inherent assumptions or alterations in the values chosen for their key parameters.

Important conclusions have been drawn from these experimental and analytical exercises. For example, they have been used to assess the wind speeds required to generate levels of turbulence that might enhance the feeding rates, growth and survivorship of fish larvae (MacKenzie \& Kiørboe 1995, Werner et al. 1995). However, as outlined above, and detailed in the Comments that follow, these assessments are based upon a number of assumptions which are, as yet, unsupported by strong empirical evidence. It seemed important to bring this to the attention of a broad readership. These comments were solicited in that spirit. 
The goal of this Comments Section was to obtain at least one comment on the turbulence issue from each of the main perspectives: physics (Osborn \& Scotti, Yamazaki), field marine/fisheries ecology (Sundby) and biology/behaviour (Strickler \& Costello for zooplankton, Browman \& Skiftesvik for ichthyoplankton) Due to space limitations, it was impossible to include every possible contributor in this exercise. Nonetheless, I hope that this Comments Section will be of value to the community, despite its inevitable imperfections. MEPS readers are invited to propose similar exercises on issues that might benefit from this kind of forum.

\section{LITERATURE CITED}

Dower J, Miller TJ, Leggett WC (in press) The role of microscale turbulence in the feeding ecology of larval fish Adv Mar Biol

Mackenzie BR, Kiorboe T (1995) Encounter rates and swimming behaviour of pause-travel and cruise larval fish predators in calm and turbulent environments. Limnol Oceanogr 40:1278-1289

Rothschild B, Osborn TR (1988) Small-scale turbulence and plankton contact rates. J Plankton Res 10:465-474

Werner FE, Perry RI, MacKenzie BR, Lough RG, Naimie CE (1995) Larval trophodynamics, turbulence, and drift on Georges Bank: a sensitivity analysis of cod and haddock Comm Meet Int Coun Explor Sea CM-ICES/Q:26

\section{Effect of turbulence on predator-prey contact rates: where do we go from here?}

\author{
Thomas Osborn ${ }^{1, *}$, Alberto Scotti ${ }^{2}$ \\ 'Department of Earth and Planelary Sciences, \\ ${ }^{2}$ Department of Mechanical Engineering, \\ The Johns Hopkins University, Baltimore, Maryland 21218, USA \\ -E-mail: osborn@jhu.edu
}

Rothschild \& Osborn (1988) put forward the concept that turbulent motions in the water increase the contact rates between predator and prey. On the local scale (i.e. for interactions between individuals), this idea is easy to accept; at a specified concentration of predator and prey organisms, any increase in relative motion is likely to increase the contact rate. Questions arise when attempts are made to quantify the calculations, and to relate the predictions to biological variables such as survival and growth rates. Can combinations of this and other effects produce a dome shaped survival rate, e.g. increasing feeding effectiveness at lower turbulent intensities which are eventually superseded by the effects of turbulence on the capture efficiency or the prey density?

To predict the effect of turbulence on the interactions of predators and prey we need progress in 4 (overlap- ping) areas: (1) knowledge of the processes, specifically the sequence of events that constitute the act of feeding and its associated activities (see Comments by Sundby and Browman \& Skiftesvik), (2) an understanding of the role of turbulent motion, swimming and other forms of relative motion on the feeding process (see Comment by Yamazaki), (3) a description of the behavior of predator and prey during feeding including both successful and unsuccessful attempts, grooming, resting, etc. (see Comments by Browman \& Skiftesvik and Strickler \& Costello), and (4) a framework to blend together the physics and biology in a fashion that permits quantitative calculations, i.e. some realistic way to model the processes.

Feeding process. What is the feeding process? Rothschild \& Osborn used 2 models for numerical calculations, the swept volume picture of Gerritsen \& Strickler (1977) and a relative diffusion model of Chandrasekhar (1943). Alternatively, Osborn (1996) suggests that turbulent diffusion of the prey towards the predator may be the mechanism by which turbulence supplies food and most strongly affects the feeding of fish larvae and copepods with feeding currents. These different mechanisms for the role of turbulence in the interaction of predator and prey are sensitive to different aspects of the turbulent field. The process cannot be correctly modeled until there are sufficient direct observations, of real feeding events, to delineate the crucial elements that a model must contain. In addition, the predators may have behavioral patterns that serve to increase their range of capture beyond their perceptual radius, such as hunting in groups or jittering around their mean position (Osborn 1996).

Present modeling treats prey not as particles, but as a continuous quantity and the predator as a point, or a sphere. This simplifies the mathematics but may not be an appropriate solution since, at low prey abundance, prey occur in discrete bundles and not in a continuous distribution. Further, the predators are laterally symmetric at best (i.e. they are not spherical) and are probably more efficient at capturing prey over a limited range of angles (see Comment by Browman \& Skiftesvik). These aspects of the problem will have to be incorporated in the modeling phase for testing against data.

Relative motion. Relative motion between predator and prey can occur for several reasons. Convergence/divergence of the water motion, swimming by either organism, sinking due to excess weight (Stokes drag), as well as effects due to the finite size and the shape of the organisms. These latter terms can become quite complicated (see Maxey \& Riley 1983 for the simple case of a small rigid sphere). Most of the calculations for the relative motion of predator and prey due to turbulent flow have assumed that the prey are 
points, that they follow the flow, and that the relative motion can be calculated from the formulae associated with homogeneous and isotropic turbulence at high Reynolds numbers (the 'Kolmogorov' formulation).

Laboratory experiments must be at appropriate values of the turbulent dissipation, $\varepsilon$, in order to scale the viscous cutoff of the turbulent field correctly with respect to the size of the predators and prey. Laboratory apparatuses are physically limited in size and, hence, the maximum length scale of the flow inside them is also limited. As a result, the Reynolds number of laboratory systems is constrained and laboratory simulations will not reach Reynolds numbers at which simple Kolmogorov formulations would be expected to hold. In such cases, the range between the energy containing eddies and the dissipation scale is not large enough to achieve the $k^{-5 / 3}$ spectral shape. Thus, while relations for the velocity difference as a function of separation, such as $\Delta u \approx(\varepsilon / \nu)^{1 / 2} \cdot r$ and $\Delta u \approx \varepsilon^{1 / 3} \cdot r^{1 / 3}$, may be appropriate at certain scales in the ocean, they cannot be applied in laboratory tanks at the same local rate of turbulent dissipation. Quantitative comparison of contact rates determined in the laboratory with those predicted using parameters derived from Kolmogorov scaling are of limited utility, even when the comparison is favorable.

Behavior. This subject gets to the heart of the problem, for the animals must have evolved in conjunction with, and in response to, the world about them. Their techniques for living must allow for the range of different situations in which they find themselves during the course of their lives, and they must exhibit appropriate responses to different stimuli. The coupled system of environment, predator and prey is highly non-linear. Being eaten is a life changing experience!

Behavioral studies using tethered copepods show a great deal about the behavior of the animals in relation to different stimuli, for both feeding and escape (J. Yen pers. comm.). However, the flow field impinging on a tethered animal is significantly different from that seen by a freely drifting animal. When tethered, the animal is sampling the velocity field in an Eulerian manner, the structure function is the Eulerian time correlation and the energy scale (and intensity) is large. A drifting animal senses the Lagrangian temporal correlation, the structure function is the Lagrangian time correlation and the energy scale (and intensity) is small. Thus, conclusions about behavioral responses to turbulence, drawn from studies on tethered animals, must be treated with some caution.

Fortunately, observational techniques are developing rapidly and it is now routine to track freely swimming animals in the laboratory (Yen \& Strickler 1996. The next step is to perform similar measurements in the ocean. For example, such observations will poten- tially differentiate between different feeding models. A copepod feeding in a swept volume mode will have a deficit of prey particles (relative to the concentration at infinity) behind itself while one feeding in a diffusion mode will have the deficit in front (Osborn 1996). The situation for fish larvae will be much more complicated to sort out (see Comment by Browman \& Skiftesvik).

A framework for calculations. We return to the initial question. What are we trying to understand? Just the role of turbulence on predator-prey contact rates or the role of turbulence on feeding, growth rates, survival, and eventually recruitment? The first question focuses on a specific aspect of the feeding process while the latter question covers many aspects of feeding and other processes. Although the latter question can presumably be attacked in a reductionist manner by understanding of all the component processes, we are nowhere near that level of understanding yet. Hence, any modeling on these big picture questions will incorporate gross parameterizations with coefficients that probably cannot be specified by, or related to, the details of the specific aspects of the problem. Hence the calculations reduce to trying to adjust parameters in the models to reproduce observations.

The modeling of predator-prey interactions, including behavioral aspects as well as the motion of the water, may well be possible. Present models for the effects of relative motion and turbulence in augmenting the contact rate between predator and prey (e.g. Gerritsen \& Strickler 1977, Rothschild \& Osborn 1988, Kiørboe \& Saiz 1995) contain substantial simplifications and the suitability for comparison with, or prediction of, the real world is problematical. However, the recent developments in observational techniques and numerical modeling of turbulent flows can be combined. The models and observations must reproduce the salient features of both the biology and the physics. With respect to the water flow, this includes not just the dissipation rate, but also the Reynolds number. Experimental systems and calculations must reproduce the turbulent field on both small scales and scales that are significantly larger than the separation of predator and prey at the moment of capture.

\section{LITERATURE CITED}

Chandrasekhar S (1943) Stochastic problems in physics and astronomy. Rev Mod Phys 15:1-89

Gerritsen J, Strickler JR (1977) Encounter probabilities and community structure in zooplankton: a mathematical model. J Fish Res Bd Can 34:73-82

Kiorboe T, Saiz E (1995) Planktivorous feeding in calm and turbulent environments, with emphasis on copepods. Mar Ecol Prog Ser 122:135-145 
Maxey MR, Riley JJ (1983) Equation of motion for a smali rigid sphere in a nonuniform flow. Phys Fluids 26:883-889

Osborn TR (1996) The role of turbulent diftusion for copepods with feeding currents. J Plankton Res 18:185-195

Rothschild BJ, Osborn TR (1988) The effect of turbulence on planktonic contact rates. J Plankton Res 10:465-474

Yen J, Strickler JR (1996) Advertisement and concealment in the plankton: what makes a copepod hydrodynamically conspicuous? Invert Biol (in press)

\section{Turbulence problems for planktonic organisms}

\section{Hidekatsu Yamazaki}

\author{
Department of Ocean Sciences, Tokyo University of Fisheries, \\ 4-5-7 Konan, Minato, Tokyo 108, Japan \\ E-mail: hide@aquarius tokyo-u-fish.ac.jp
}

The turbulent velocity spectrum is comprised of 2 components: an uncorrelated and a correlated velocity field. The correlated field can be considered as follows When 2 separated points in a turbulent field move in parallel, the relative speed of these points is zero. In order for one point to approach the other, one of the points must actively move toward the other. In this scenario, no increase in the encounter probability between prey and predator is expected. On the other hand, when a flow field between 2 separated points is uncorrelated, passive particles at these locations can move independently. The encounter rates between prey and predator can increase only due to this portion of the turbulent velocity spectrum. The root-meansquare turbulent velocity derived in Rothschild \& Osborn (1988) refers to the uncorrelated part of the turbulent velocity spectrum. This is a very important aspect of the theoretical development surrounding turbulence, and one should not overlook the assumption (j.e. the exclusion of the correlated component).

Why do 2 parts, namely correlated and uncorrelated, exist in a turbulent velocity field? Although turbulence has been treated as a nearly random flow, the flow must obey dynamical constraints (the Navier-Stokes equations) and satisfy the kinematic condition (the continuity equation). These constraints generate temporal and spatial patterns in the turbulent flow field. These patterns can remain in space for a finite amount of time, and contribute to the correlated component of the turbulent flow field. The pattern is called a coherent structure. Turbulence is not a complete chaos; in fact, it has an 'order'!

In general, when the ambient flow field in which prey and predator find themselves is turbulent, the uncorrelated component of the turbulent velocity field increases the encounter rates. As the distance between prey and predator increases, the uncorrelated component of the turbulent velocity increases. Thus, the larger the distance is, the larger the encounter rate is However, the predator requires a large detection distance, $d$, in order to take maximum advantage of the increase in the uncorrelated turbulent velocity.

What value should one consider to be an appropriate choice for $d$ in the encounter rates problem? This cannot be answered from a purely physical standpoint. We must also consider the biological aspects of the question (see Comments by Browman \& Skiftesvik and Strickler \& Costello). Since I have been working on the turbulence-related encounter problem for zooplankton, I will consider these predators, whose total length scale is $1 \mathrm{~mm}$, as my target organism in the following discussion. However, the discussion can be extended to ichthyoplankton.

As a rule of thumb, a detection distance for zooplankton is roughly a few body lengths (Haury \& Yamazaki 1995). Therefore, for a zooplankter of $1 \mathrm{~mm}$ body length, a maximum $d$ is no more than $5 \mathrm{~mm}$. In order to consider turbulence effects on this biological length scale, I will consider 2 levels of dissipation rates to derive the relevant physical scales. For the first, I will take a typical turbulent patch in a seasonal thermocline whose dissipation rate is $\varepsilon_{1}=10^{-8} \mathrm{~W} \mathrm{~kg}^{-1}$. As the second level, 1 will choose $\varepsilon_{2}=10^{-4} \mathrm{~W} \mathrm{~kg}^{-1}$, representing a near ocean surface turbulence. The Kolmogorov scale is used to separate a viscosity dominated flow regime from an inertia dominated flow regime. When a flow scale becomes smaller than the Kolmogorov scale, the flow field is quite viscous, and correlated with each other The Kolmogorov scale for $\varepsilon_{1}$ is $3.2 \mathrm{~mm}$, and $\varepsilon_{2}$ is $0.32 \mathrm{~mm}$. Hence, $d$ is nearly the same for the thermocline case, but is about 10 times greater than that of the surface turbulence case. This implies that the near surface turbulence may significantly increase the encounter rates between prey and predator. On the other hand, $d$ for the thermocline value is almost the same as the Kolmogorov scale, thus no significant increase in the encounter rates is expected in this regime.

Even if strong winds induce high turbulence near the surface, the level of turbulence drops almost exponentially. Within a short distance - on the order of $10 \mathrm{~m}-$ the dissipation rate can decrease to at least 100 times smaller than the near surface value. Therefore, a significant increase in the encounter rates between prey and predator due to turbulence is likely limited to a thin layer near the sea surface.

Although the rest of the turbulent regime beneath the surface turbulent layer may not significantly affect planktonic encounter rates, turbulence may play a role 
in other aspects of zooplankton ecology. The encounter rate calculation is based on the uncorrelated component of the turbulent velocity field. A large fraction of the turbulent velocity field is correlated, particularly when the distance, $d$, approaches the Kolmogorov scale. We have not paid much attention to how the correlated component of the flow field may affect plankton ecology (Yamazaki 1993).

Despite the fact that we do not know much about the nature of coherent structures, some progress has been made through direct numerical simulation (DNS) of Navier-Stokes equations (Hussain 1986) Squires \& Yamazaki (1995) used DNS to follow marine particles in an isotropic turbulent flow. A total of 165888 particles were placed uniformly at their initial locations. Due to the excess density of the marine particle relative to the ambient fluid, i.e. a particle:ambient fluid density ratio of 1.06 , the particles become preferentially concentrated in regions of low vorticity or high strain rate. The peak number density is 40 times larger than the global mean value. If the flow is completely random, this kind of local aggregation cannot take place.

DNS of zooplankton in isotropic turbulence shows that a swimming behavior model based on the local flow strain rate can take advantage of flow structures to sustain the mean vertical position of a negatively buoyant particle (K. D. Squires pers. comm.). The model swimming pattern is controlled by the local rate of velocity strains, and is aligned with the intermdeiate eigenvector by taking the direction of gravity into account. Since mechanical receptors can detect velocity strain much easier than absolute velocity, the swimming behavior model is a realistic mechanism which conserves biological energy to sustain a negatively buoyant body in a water column.

Since zooplankton have evolved behavioral adaptations to flow patterns, the issue of coherent structures is important. We must realize that the encounter rates problem is not the only effect of turbulence on zooplankton ecology of which we need to be aware.

\section{LITERATURE CITED}

Haury LR, Yamazak1 H (1995) The dichotomy of scales in the perception and aggregation behavior of zooplankton. J Plankton Res 17:191-197

Hussain AKMF (1986) Coherent structures and turbulence. J Fluid Mech 173:303-356

Rothschild BJ, Osborn TR (1988) The effect of turbulence on planktonic contact rates. J Plankton Res 10:465-474

Squires KD, Yamazaki H (1995) Preferential concentration of marine turbulence. Deep Sea Res 42:1989-2004

Yamazaki H (1993) Lagrangian study of planktonic organisms: perspectives. Bull Mar Sci 53:265-278

\section{Turbulence-induced contact rates in plankton: the issue of scales}

\author{
Svein Sundby \\ Institute of Marine Research, PO Box 1870 Nordnes, \\ N-5024 Bergen, Norway \\ E-mal: svein.sundbyemr.no
}

A rapidly increasing quantity of literature has evolved in the wake of the theory developed by Rothschild \& Osborn (1988) on the effects of turbulence on plankton contact rates. Although their theory focused on small scales of isotropic turbulence, the general concepts that they proposed are not limited to those scales.

The choice of scale is essential to assessing the effects of turbulence on the interactions between particles in the plankton. Choosing the appropriate scale is dependent upon the relative motion of predator and prey and on the distance between them. However, the fact that turbulence in a natural system occurs simultaneously at all length scales means there is no trivial answer to the question of which of these scales contributes to enhancement of the contact rate between particles. A complete mathematical formulation for this part of the theory on turbulence-induced contact rate is still lacking. However, it follows from physical reasoning that the relevant turbulent length scales are linked to the separation distance, $I$, between interacting particles.

Turbulent diffusion (or spreading) of particles is the result of the same physical process that causes contact between them, and it is a basic property of turbulent diffusion that the turbulent diffusivity coefficient increases as the size of the diffusing cloud of particles increases (e.g. Okubo 1978). This is so because larger and larger turbulent eddies will take part in the turbulent mixing as the size of the cloud increases. Rothschild \& Osborn (1988) developed an expression for the root-mean-square turbulent velocity: $w=1.9(\varepsilon \cdot d)^{1 / 3}$ (where $\varepsilon$ is turbulent kinetic energy dissipation rate and $d$ is the length scale of the uncorrelated velocity fluctuation). This expression was used to calculate the velocity component of the turbulence-induced contact rate. Since the turbulent eddy diffusivity coefficient is linked to the size of the particle cloud, then, by analogy, the turbulence-induced contact rate between particles is linked to the separation distance between them. Hence, the length scale, $d$ is proportional to the separation distance $r, d \sim r$, where $r=c^{-1 / 3}$ is the mean deterministic separation distance between particles at concentration $c$.

As an approximation for the relevant turbulent scale, d, in the equation above, Sundby \& Fossum (1990), Sundby et al. (1994) and Sundby (1995) used $d=r$ in their calculations. More correctly, however, it is really 
the sum of all turbulent length scales, $d_{1}$ less than $r$ which contribute to enhancement of the contact rate, because all turbulent scales less than that of the separation distance will change the relative position between the particles and hence contribute to contact. But, since turbulent velocities are larger at the larger length scales, those turbulent velocities generated on the larger length scales contribute more toward increasing the contact rate than do the velocities of the smaller turbulent length scales. Therefore, the error introduced by using $d=r$ is arguably moderate compared to the fact that the contact rate was evaluated over a relatively large range of particle concentrations, from 1 to 50 nauplii $l^{-1}$, with corresponding separation distances from 10 to $3 \mathrm{~cm}$.

Turbulence at scales considerably larger than the particle separation distance will not, however, contribute to enhancement of the contact rate, since the turbulent 'cells' on these larger length scales will only contribute to the moving around of smaller parcels of fluid without rearranging the positions of the particles within these smaller fluid parcels. The process of turbulence-enhanced contact rate, or the rate of collision between particles, is a physical process which will influence particles in the same way whether they happen to be large or small, biotic or abiotic, dead or alive. For a predator to successfully ingest a prey, however, there are a set of additional biotic processes which must be considered after the prey is located. Here, the reactive distance, $R$, the maximum distance at which a given prey can be perceived, becomes relevant with respect to the turbulent length scale $d$. However, $R$ is independent of the preceding physical encounter process, although it enters the calculation of the volume searched by a fish larva as a constant.

Kiørboe \& MacKenzie (1995), Kiørboe \& Saiz (1995) and MacKenzie \& Kiorboe (1995) propose that the predator's reactive distance, $R$, and not the particle separation distance, $r$, is the relevant scale over which to evalute the effects of turbulence on encounter and ingestion. Following the above reasoning, this proposal appears to be based upon the assumption that turbulence only affects the components of the predation cycle that follow prey location. Successful ingestion of prey, however, consists of at least 4 consecutive processes:

(1) The time required to search for prey prior to encounter or contact. The search process is the most time consuming part of the predation cycle for many predators, particularly carnivorous plankton (O'Brien et al. 1990). For any kind of interacting vehicles, such as navy vessels (Koopman 1956) or combat airplanes (Kohlas 1967), it is the relative velocity between the vehicles which determines the change in position between them and, hence, the probability of en- counter. The direct analogy in the animal world is the relative movement between a predator and its prey. For plankton, turbulence adds to the change of relative position between them; and, here, the mean separation distance between the predator and prey is the key parameter determining the scale of relative motion. Hence, and as argued above, during this part of the predation cycle, $d \sim r$

(2) Prey location time. This is usually a relatively short interval of time, compared to the search period, during which the predator scans its visual perceptual field for prey and makes the decision to attack or ignore it. Once a prey item has been located, the distance between the predator and prey can be no greater than the reactive distance, $R$, of the predator. Now, the situation is linked to the one specific prey which has been located, whereas in the search process above the situation was linked to all the surrounding potential prey. The turbulent length scales which in this situation contribute to changes in the relative distance between predator and prey are now all length scales equal to and smaller than $R$. Hence, $d \sim R$.

(3) The time of pursuit and attack by the predator and/or escape by the prey. This time interval, during which the attack occurs, is also quite short. The prey is still no farther from the predator than the reaction distance, $R$, and therefore the turbulent 'cells' which contribute to changes in the relative distance in this situation are still, as in the situation above, those of length scales equal to or smaller than $R$. Hence, $d \sim R$.

(4) The time required to ingest prey. Predator and prey are no longer separated and the turbulence of the ambient water can, of course, no longer contribute to changes in the relative distance between them. I therefore tend to assume that it is unlikely that turbulence has much affect on this last component of the predation cycle. It could be speculated that very strong accelerations induced by turbulence might cause regurgitation of the prey, but what turbulent scale this might involve is unclear.

When Kiørboe \& MacKenzie (1995), Kiørboe \& Saiz (1995) and MacKenzie \& Kiørboe (1995) only consider the time intervals (2) and (3) as relevant for turbulenceinduced encounter rate they neglect the most important and longest time interval of the predation cycle prey search. However, they also contradict the results of Mackenzie et al. (1994), who proposed a domeshaped relationship between turbulence and larval fish ingestion rates. Mackenzie et al. (1994) found, conversely, that the effect of turbulence on the scale of reaction distance, $R$, contributed to a decrease in the encounter rate, not to an increase in it

Kiørboe \& MacKenzie (1995) cite Evans (1989) to support their choice of reactive distance as the correct scale to consider. However, Evans (1989) simply states, 
without any further argument, that the appropriate length scale for the uncorrelated component of the turbulent velocity is precisely the encounter radius, $R$. Kiørboe \& Saiz (1995) also cite Delichatsios \& Probstein (1975) and Hill et al. (1992) to justify the idea that only the smallest turbulent scales are relevant in plankton encounters. However, all of the authors cited in support of this assertion considered closely spaced particles at concentrations several orders of magnitude higher than the abundances which would be realistic, and of relevance, for interactions between larval fish and their prey. Delichatsios \& Probstein (1975) and Hill et al. (1992) considered only the smallest turbulent scales, around the Kolmogorov scale, in their work on coagulation of very small particles and, hence, their conclusions confirm that the turbulence-induced contact rate is linked to the separation distance between the particles and not to the reaction distance.

There are many implications of relative motion with respect to plankton predator-prey interactions, some of which are linked to behavioral and biological responses (e.g Rosenthal \& Hempel 1970, Browman \& O'Brien 1992; see Comments by Browman \& Skiftesvik and Strickler \& Costello), others of which are related to physical processes. The way in which the cascade of turbulent scales interact depends on the specific process of plankton interaction which is considered.

\section{LITERATURE CITED}

Browman HI, O'Brien WJ (1992) The ontogeny of search behaviour in the white crappie Pomoxis annulars. Environ Biol Fish 34:181-195

Delichatsios MA, Probstein RF (1975) Coagulation in turbulent flow: theory and experiment. J Colloid Interface Sci 51:394-405

Evans G (1989) The encounter speed of moving predator and prey. J Plankton Res 11:415-417

Hill PS, Nowell ARM, Jumars PA (1992) Encounter rate by turbulent shear of particles simlar in diameter to the Kolmogorov scale. J Mar Res 50:643-668

Kırboe T, Mackenzie B (1995) Turbulence-enhanced prey encounter rates in larval fish: effects of spatial scale, larval behaviour and size. J Plankton Res 17:2319-2331

Kiorboe T, Saiz E (1995) Planktivorous feeding in calm and turbulent environments with emphasis on copepods. Mar Ecol Prog Ser 122:135-145

Kohlas J (1967) Simulation von Luftkampfen. PhD thesis, Universität Zürich

Koopman BO (1956) The theory of search. I. Kinematic bases. Oper Res 4:324-346

MacKenzie BR, Kiørboe T (1995) Encounter rates and swimming behaviour of pause-travel and 'cruise' larval fish predators in calm and turbulent environments. Limnol Oceanogr 40:1278-1289

MacKenzie BR, Miller TJ, Cyr S, Leggett WC (1994) Evidence for a dome shaped relationship between turbulence and larval fish ingestion rates. Limnol Oceanogr 39:1790-1799

O'Brien WJ, Browman HI, Evans Bl (1990) Search strategies of foraging animals. Am Sci 78:152-160
Okubo A (1978) Horizontal dispersion and cratical scales for phytoplankton patches. In: Steele $\mathrm{JH}$ (ed) Spatial pattern in plankton communities. Plenum Press, New York, $p$ $21-42$

Rosenthal H, Hempel G (1970) Experimental studies in feeding and food requirements of herring larvae (Clupea harengus L.). In: Steele JH (ed) Marine food chains. University of California Press, Berkeley, p 344-364

Rothschild BJ, Osborn TR (1988) Small-scale turbulence and plankton contact rates. J Plankton Res 10:465-474

Sundby $S$ (1995) Wind climate and foraging of larval and juvenile Arcto-Norwegian cod. Can Spec Publ Fish Aquat Sci 121:405-415

Sundby S, Ellertsen B, Fossum P (1994) Encounter rates between first-feeding cod larvae and their prey during moderate to strong turbulent mixing. ICES Mar Scl Symp 198:393-405

Sundby S, Fossum P (1990) Feeding conditions of ArctoNorweglan cod larvae compared to the Rothschild-Osborn theory on small-scale turbulence and plankton contact rates. J Plankton Res 12:1153-1162

\section{Calanoid copepod behavior in turbulent flows}

\author{
J. Rudi Strickler ${ }^{1,}$, John H. Costello
}

${ }^{1}$ Center ior Great Lakes Studies, University of Wisconsin - Milwaukee, 600 E. Greenfield Ave., Milwaukee, Wisconsin 53204, USA

${ }^{2}$ Biology Department, Providence College, Providence, Rhode Island 02918, USA

-E-mail: jrs@csd.uwm.edu

'...in solving ecological problems we are concerned with what animals do in their capacity as whole, living animals, not as dead animals or as a series of parts of animals. We have next to study the circumstances under which they do these things, and, most important of all, the limiting factors which prevent them from doing other things. By solving these questions it is possible to discover the reasons for the distribution and numbers of different animals in nature.' (Elton 1927).

Since the publication of Rothschild \& Osborn's (1988) seminal paper, the influence of small scale turbulence on planktonic encounter rates and, therefore, trophic interactions, has been debated in biological oceanography. Whereas the original model assumed that predators and prey were inanimate points in 3-dimensional space, more recently Osborn (1996, p. 194) concluded that 'treating predators and prey as simple points is not adequate for quantitative predictions'. Clearly, the elevated encounter rates between plankters in a turbulent flow will not result in enhanced trophic exchange unless the zooplankters adapt behaviorally to exploit the high encounter rates. The behavioral response of copepods to turbulence has been the central focus of our work 
Our approach has relied on 2 basic premises. First, that copepods are organisms with complex sensory and behavioral capabilities. Any response to the environment, such as turbulence, is mediated through their behavior. Second, that we can measure and characterize these behavior patterns by direct observation of whole animals under carefully controlled environmental conditions. Our methods are based on the previous work of Cowles \& Strickler (1983) who observed the behavior of tethered Centropages typicus (a coastal calanoid copepod) exposed to different species and concentrations of phytoplankton. That research established that time allocated to slow swimming was a quantifiable variable representing foraging effort. Slow swimming utilizes only the feeding appendages whereas rapid swimming, an escape response, also involves movements of the first antennae and the urosome.

Our knowledge of copepod responses to turbulence has developed through several related studies. Costello et al. (1990) and Marrasé et al. (1990) exposed a female Centropages hamatus to 6 different environmental conditions: 2 food concentrations each with pre-turbulent, turbulent and post-turbulent periods The food concentrations were chosen so that the high food/pre-turbulent condition resulted in the same encounter rate as the low food/turbulent conditions. The encounter rates were quantified by direct observation and the energy dissipation rates were determined using aigal tracks (Dickey \& Mellor 1980). The results showed clearly that the 3 common functional response curves (Holling 1965) did not adequately describe the feeding behavior at different encounter rates. Foraging effort (slow swimming) increased during turbulence However, the experience of a turbulent condition affected foraging during the subsequent non-turbulent period (Figs. $2 \& 4$ in Costello et al. 1990). Subsequent experiments supported this finding and extended it to conditions of alternating turbulent and non-turbuient flow of different durations (Hwang \& Strickler 1994, Hwang et al. 1994). As with the previous studies of Costello et al. (1990) and Marrasé et al. (1990), when exposed to cycles of non-turbulent followed by turbulent conditions, the copepod C. hamatus initially responded with numerous escape reactions and increased foraging behavior. However, after turbulent followed by non-turbulent conditions were repeated for several consecutive cycles, these 2 behaviors followed distinctly different patterns. Foraging effort during exposure to turbulent conditions increased, and, after 2 cycles, remained at high levels throughout non-turbulent periods. In contrast, escape behavior was habituated rapidly during each turbulent period and dishabituated during each non-turbulent period. Sensitivity to mechanical stimuli (defined as the particle velocity necessary to initiate an escape response) declined during this habituation process (Hwang \& Strickler 1994). The adaptive significance of this behavioral response pattern may lie in the ability of $C$. hamatus to respond to intermittent turbulence by maximizing foraging during periods of enhanced encounter rates while minimizing the energetic expense of unnecessary escape responses via escape response habituation.

The relevance of our laboratory work to field conditions remains an important area of research. The experiments described above used tethered Centropages hamatus females exposed to turbulence with $\varepsilon$ values of 0.05 to $0.15 \mathrm{~cm}^{2} \mathrm{~s}^{-3}$ (Marrasé et al. 1990). These values fall within the range characterizing coastal waters and well-mixed oceanic upper layers (Granata \& Dickey 1991; see Comment by Yamazaki). However, our experiments were conducted under artificial conditions because a tcthered copepod cannot move relative to the fluid motions around it (see Comment by Osborn \& Scotti). Under natural conditions, fluid flow at the millimeter length scale is predominantly viscous (Lazier \& Mann 1989). Under these conditions, copepods may simply be entrained within the smallest eddies (the diameter of which is described by the Kolmogorov length scale) and may not be able to detect the presence of turbulence. In this case, the behavior of copepods in natural turbulent flows might not differ from that of copepods in non-turbulent flows, rendering the topic of turbulence a non-issue for copepod feeding. However, recent studies cast doubt on the assumption that viscosity dominates small scale particle interactions near the Kolmogorov scale (Hill et al. 1992; but see Comments by Osborn \& Scotti, Sundby, and Yamazaki). Further, turbulence may affect copepod grazing even if the transition to turbulent diffusion occurs at length scales longer than the copepod and its feeding current (Osborn 1996). These studies highlight the need for more extensive in situ measurements and direct observations of copepod feeding.

A second major area of copepod ecology affected by turbulence involves inter- and intraspecific communication. Recent observations of calanoid copepods demonstrate the importance of female scent trails for mating. Males encountering female scent trails accelerate swimming and cover distances up to $10 \mathrm{~cm}$ in order to overtake and mate with females (Colin 1995, Strickler \& Hwang in press). Additionally, species specific wakes are used for inter-and intraspecific signaling (Yen \& Strickler 1996). The length scales over which these processes occur are well within the length scales affected by turbulence and could be decisively influenced by the character of turbulent flows in nature. 
Needless to say, to date we have touched upon only the tip of an iceberg Much more research of this nature will be needed if we want to make our models more realistic, albeit more complex.

\section{LITERATURE CITED}

Colin SP (1995) A kınematic analysis of trail-following in Temora longicornis and four other copepods: how the male finds his mate. MSc thesis. State University of New York, Stony Brook

Costello JH, Marrasé C, Strickler JR, Zeller R, Freise AJ, Trager G (1990) Grazing in a turbulent environment: I. Behavioral response of a calanoid copepod, Centropages hamatus. Proc Nat Acad Sci USA 87:1648-1652

Cowles TJ, Strickler JR (1983) Characterization of feeding activity patterns in the planktonic copepod cintropages typicus Kroyer, under various food conditions. Limnol Oceanogr 28:106-115

Dickey TD, Mellor GL (1980) Decaying turbulence in neutral and stratified flunds. J Fluid Mech 99:13-31

Elton C (1927) Animal ecology. Sidgwick and Jackson, London

Granata TC, Dickey TD (1991) The fluid mechanics of copepod feeding in a turbulent flow: a theoretical approach. Prog Oceanogr 26:243-261

Hill PS, Nowell ARM, Jumars PA (1992) Encounter rate by turbulent shear of particles similar in diameter to the Kolmogorov scale. J Mar Res 50:643-668

Holling CS (1965) The functional response of predators to prey density and its role in mimicry and population regulation. Mem Entomol Soc Can 45:1-60

Hwang JS (1991) Behavioral responses and their role in prey/predator interactions of a calanoid copepod, Centropages hamatus, under variable hydrodynamic condituons. PhD thesis, Boston University

Hwang JS, Costello JH, Strickler JR (1994) Copepod grazing in turbulent flow: elevated foraging behavior and habituation of escape responses. J Plankton Res 16:421-431

Hwang JS, Strickler JR (1994) Effects of periodic turbulent events upon mechanoreception and escape responses of a calanoid copepod, Centropages hamatus. Bull Plankton Soc Japan 41:117-130

Lazier JRN, Mann KH (1989) Turbulence and diffusive layers around small organisms. Deep Sea Res 36:1721-1733

Marrasé C, Costello JH, Granata T, Strickler JR (1990) Grazing in a turbulent environment: II. Energy dissipation, encounter rates and efficacy of feeding currents in Centropages hamatus. Proc Nat Acad Sci USA 87: $1653-1657$

Osborn TR (1996) The role of turbulent diffusion for copepods with feeding currents. J Plankton Res 18:185-195

Rothschild BJ, Osborn TR (1988) The effect of turbulence on planktonic contact rates. J Plankton Res 10:464-475

Strickler JR. Hwang JS (in press) Matched spatial filters in long working distance microscopy of phase objects. In: Cheng PC, Hwang PP, Wu JL, Wang G, Kim H (eds) Focus on modern microscopy. World Scientific Publishing Inc, River Edge, NJ

Yen J, Strickler JR (1996) Advertisement and concealment in the plankton: what makes a copepod hydrodynamically conspicuous? Invert Biol (in press)

\section{Effects of turbulence on the predation cycle of fish larvae: comments on some of the issues}

\author{
Howard I. Browman ${ }^{1,}$, Anne Berit Skiftesvik ${ }^{2}$ \\ ${ }^{1}$ Department of Fisheries and Oceans Canada, \\ Maurice Lamontagne Institute, Ocean Productivity Division, \\ PO Box 1000, Mont-Joli, Quebec, Canada G5H 3 Z4 \\ 'Institute of Marine Research, Aquaculture Centre, Austevoll \\ Aquaculture Research Station, N-5392 Slorebo, Norway \\ -E-mail: h_browman@qc.dfo.ca
}

Turbulence-induced changes in ichthyoplanktonzooplankton (I-Z) contact rate (as a function of absolute prey abundance) have been proposed as an important factor in determining prey ingestion rate and, therefore, growth and mortality rates in fish larvae (reviewed by Dower et al. in press). The role of turbulence in the feeding ecology of fish larvae has been evaluated through various combinations of laboratory, field and analytical exercises (e.g. Kiørboe \& MacKenzie 1995, MacKenzie \& Kiørboe 1995, Sundby 1995). As outlined in the introduction to this Comments Section, each of these approaches is associated with a series of inter-related assumptions which have only rarely been subjected to rigorous scrutiny (although see Mackenzie et al. 1994, Mackenzie \& Kiørboe 1995, Dower et al. in press).

Here, we comment upon some of the issues surrounding turbulence-induced effects on the predation cycle of fish larvae.

(1) All of the components of the predation cyclesearch, location, pursuit or abort (i.e. the choice not to pursue a located prey), attack and capture, ingestion and retention - might be affected by turbulence. However, most of the attention has focussed on the rate of prey encounter (or contact) which, from the behavioural perspective, is operationally equivalent to prey location. Little effort, particularly in the form of empirical observations, has yet been devoted to evaluations of the effects of turbulence on any other component of the predation cycle. In addition, and as noted in the Comment by Sundby, clear differentiation amongst the possible effects of turbulence on each component of the predation cycle has been lacking in many studies.

(2) For small planktivores, which scan the water near to them for discrete and sparsely distributed prey items, search is the most time consuming component of the predation cycle (O'Brien et al. 1990). To illustrate: the volume of water contained in the visual perceptual field (VPF) of a 6 to $10 \mathrm{~mm}$ fish larva is approximately 0.8 to $1.0 \mathrm{ml}$. At an absolute prey abundance of $50 \mathrm{l}^{-1}$, there would be only 0.04 to 0.05 prey items within the VPF at any given moment (the number of prey per VPF is the visual abundance, VA. Note that the VA is, in this 
case, 3 orders of magnitude less than the absolute abundance). Clearly, fish larvae must scan a significant amount of water in order to locate a prey item, and that takes a great deal of time.

Turbulence may reduce search time by bringing more prey items into the larva's VPF than would have been present in static water; that is, by increasing the VA. However, for the reasons outlined below, an increase in the VA does not necessarily equate to an increase in the rate of prey location, nor ingestion.

(3) The geometry of a fish larva's VPF determines its volume and, therefore, the number of prey that are contained within it. As a result, VPF geometry is central to analytical and/or numerical evaluations of the effects of turbulence on $\mathrm{I}-\mathrm{Z}$ interactions.

The VPF geometry most often employed in analytical models is a sphere or a hemisphere (e.g. Mackenzie et al. 1994, Muelbert et al. 1994, Jenkinson 1995, Mackenz1e \& Kiorboe 1995). Several authors have recognized that this characterization of the VPF is inaccurate (e.g. Mackenzie et al. 1994, Dower et al. in press), yet there has been little discussion of alternate VPF geometries nor of the effect that they might have on model predictions. Although VPF geometries have been reported for only a very small number of species, none are spherical or hemispherical. Rather, VPFs most often resemble a piece of pie with maximum lateral angles approximating 80 to $120^{\circ}$ to each side of the fish's midline (Arnold \& Holford 1989, Browman \& O'Brien 1992a, b, Coughlin 1993). In the vertical dimension, the VPF is shallow at the triangular pie slice's point (at the fish's eyes), thickens towards its outer edge (the pie's crust), and usually includes more of the volume of water above the larva's midline than below (see Browman et al. 1990, Fig. 1 therein). Several important points follow from this.

(a) The volume of water contained in a shallow piewedge (and scanned for prey) is approximately 4.5 times less than that in a hemisphere of equivalent radius. Thus, at any given absolute prey abundance, the VA for a pie-wedge VPF is 4.5 times less than the VA for a hemispherical VPF

(b) The probability of a prey item being flushed into a pie-wedge VPF by turbulent water motion is significantly less than that for a hemispherical VPF This is based mainly upon the difference in geometry and is relatively independent of volume.

(c) The probability of a prey item entering a hemispherical VPF is the same for any incident vector. For a pie-wedge VPF, this probability will vary with the angle at which the prey is being flushed through the VPF's volume. To visualize the significance of this it may help to imagine a prey item moving through a piewedge along the vertical vs the horizontal. axes and to compare the length of its trajectory within the VPF to that for a hemispherical VPF; for most angles of incidence, the trajectories are much shorter for a piewedge.

(d) For asymmetrical VPFs, quantifying the probabilities just defined will not be trivial.

(e) Since scanning the VPF takes time, a prey item which is entrained in a turbulent eddy may move through the larva's VPF in less time than it takes to scan the entire volume. Thus, just because a prey item is within the VPF at any given instant does not mean that it will be located (i.e. contacted, or encountered) Restated, although turbulence affects the probability of a prey item entering the VPF, it also affects the probability of it remaining there long enough to be located. Further, even if the prey does remain within the VPF long enough to be located, the larva needs still more time to pursue, attack and ingest it

Unfortunately, scan time is a variable that has not bccn measured for fish larvae, although it may be related to the duration of stationary periods, at least in some species (O'Brien et al. 1990, Browman \& O'Brien 1992a, b, Mackenzie \& Kiørboe 1995).

(f) A critical issue in assessing the effects of turbulence on I-Z interactions is the choice of $d_{1}$ the distance between 2 points for which the velocity difference due to fluid motion $(w)$ is to be calculated. Some authors (e.g. Kiorboe \& Mackenzie 1995) argue that $d$ should be the maximum distance at which the predator can perceive prey, the reaction distance (RD). The rationale for this proposal is based upon the assertion that the turbulence-generated prey location rate is equal to the rate at which particles arrive at the surface of the larva's VPF (Kiørboe \& MacKenzie 1995). However, for any asymmetrical VPF geometry (including a hemisphere), the use of $\mathrm{RD}$ in this context is not tenable since the surface of the larva's VPF and, therefore, $d$, will be a variable distance away (and often much less than the maximum RD value) and dependent upon the prey's angle of incidence.

(g) Clearly, the results of any analytical or numerical evaluation of the effects of turbulence on I-Z interactions will be strongly affected by the choice of VPF geometry. Based upon volumes and geometries alone, the outcomes could be different by at least 1 order. of magnitude.

(4) VPF geometries and volumes are estimated from maximum prey location distances (Browman et al. 1990). For non-hemispherical VPFs, these maximum distances vary significantly with the relative angle at which the prey was located and are typically greatest straight in front of the larva and on the same vertical plane (Browman et al. 1990, Browman \& O'Brien. $1992 a$, b). However, at any given angle, prey location events within a larva's VPF are distributed from very close to the fish out to the maximum location distance 
(i.e. the RD). As an aside: although not yet widely known, this appears to be true for both pause-travel and cruise searchers (Arnold \& Holford 1989, authors' unpubl. obs.).

It follows from these observations that using the RD to calculate the VPF's volume will yield an overestimate, even if the geometry is correct. Using the frequency distribution of prey location distances, or the median location distance, and not the RD, would be more accurate

Since both the geometry and the volume of the VPF are at the heart of the encounter rate calculation [e.g. see Eq. (4) in Kiørboe \& Mackenzie 1995 or Eqs. (1a) \& (1b) in Mackenzie \& Kiørboe 1995], and since RD enters these equations as a squared term, clarifying the manner in which this variable is applied, and using accurate values, is essential.

(5) Behavioural responses to turbulence have been observed in copepods (e.g. Marrasé et al. 1990, Hwang et al. 1994, Saiz 1994; see Comment by Strickler \& Costello). In fish larvae, such behavioural responses might include turbulence-induced changes in various components of the predation cycle, for example, swimming speed, stop frequencies and durations, move distances, turn angles, the geometry and volume of the VPF, the time required to scan the VPF for prey, or the proportion of attacks initiated that are aborted. We are aware of only 1 study that has examined such effects in fish larvae (MacKenzie \& Kiørboe 1995). These authors quantified larval attack posture rate at different prey abundances and under turbulent vs non-turbulent conditions. For any given prey abundance, the static water treatment was considered as the control/base-line against which the effect of turbulence was evaluated However, since the possibility of an inherent behavioural response to turbulence cannot be excluded, a more appropriate control would be to measure the larva's response to turbulence in the absence of prey and to use this as a base-line against which to evaluate the responses at higher prey abundances. The choice of control in such experiments is critical.

(6) The rate at which fish larvae take up attack postures has been considered a reasonable proxy for prey location rate (Mackenzie \& Kiørboe 1995). In this context, it is worth noting that fish larvae often take up attack postures without ever actually striking at a prey item. These 'aborted' attacks can comprise $50 \%$ or more of all the observed attack postures (Browman \& O'Brien 1992b). Since turbulence will constantly be moving prey items into and out of the VPF, it seems reasonable to predict that the proportion of all prey attacks that are aborted will be related to the level of turbulence. If this is the case, determining the relationships between the rates of prey location, attack and ingestion under turbulent conditions will be difficult.
(7) Several recent analytical models use components of the larval prey search pattern (duration of stationary pauses and moves, move distances, stationary pause frequencies, etc.) as model inputs (Mackenzie \& Kiorboe 1995, Kiørboe \& MacKenzie 1995, reviewed by Dower et al. in press). Model output will be strongly affected by the values entered for these variables, although this has not been carefully assessed (however, see Mackenzie et al. 1994, Jenkinson 1995, MacKenzie \& Kiørboe 1995). Values for these parameters are drawn from a very limited body of literature and, so, suffer from the same problems as defined above for RDs. Further, the great majority of data on these variables has been generated in static water conditions. Since they are all likely to vary significantly under turbulent conditions, use of these data in analytical models should be undertaken with some caution. We propose that it would be more appropriate to use median values or frequency distributions, and not means, in parameterizing these variables.

(8) Some attempt has been made to evaluate the different effect that turbulence might have on pausetravel (PT) vs 'cruise' searching fish larvae (Kiørboe \& Mackenzie 1995, MacKenzie \& Kiorboe 1995). These analytical exercises have modelled 'cruise' searchers as scanning while swimming and perceiving prey as they pass across the boundary of the VPF (the classical characterization of Rosenthal \& Hempel 1970). However, there is no empirical evidence to support this characterization of 'cruise' searching and recent evidence indicates that it may be incorrect (Arnold \& Holford 1989, authors' unpubl. obs.)

Search strategies have only been carefully evaluated for a very small number of fishes (O'Brien et al. 1990). Until more such characterizations are available, particularly for search patterns other than PT, the value of analytical exercises such as those referred to above will be limited.

(9) Turbulence may affect the contact rate between fish larvae and those planktonic organisms that feed on them: the role that turbulence plays in altering the predation rate on fish larvae requires more attention than it has received to date.

To summarize: empirical evidence to support the behavioural characterizations and assumptions made in many analytical evaluations of the effects of turbulence on the feeding ecology of fish larvae is sparse Until the issues discussed above can be addressed, studies on the role of turbulence on I-Z interactions, or on fish larval growth or survivorship, should be interpreted cautiously. Laboratory experiments and/or field measurements that attempt to evaluate the effect of turbulence on growth rate or survivorship (e.g. Sundby et al. 1994, Landry et al. 1995), without regard to the mechanism, may be the most appropriate approach at 
this time. Such observations may serve to tell us whether turbulence is, in fact, so important in I-Z interactions that it merits the significant effort that will be required to determine and model its mechanism of action.

Acknowledgements. We thank John Dower, Peter Galbraith, Thomas Miller, Patrick Ouellet, Karin Pittman, Roberto Racca, Jeffrey Runge and Svein Sundby for their enthusiastic discussion of these issues and for their comments on various drafts of the text.

\section{LITERATURE CITED}

Arnold GP, Holford BH (1989) The reactive perceptive field of the larval plaice (Pleuronectes platessa L.): a three-dimensional analysis of visual feeding. Rapp P-V Reun Cons Int Explor Mer 191:474

Browman HI, Gordon WC, Evans BI, O'Brien WJ (1990) Correlation between histological and behavioral measures of visual acuity in a zooplanktivorous fish, the white crappie (Pomoxis annularis). Br Behdv Evolut 35:85-97

Browman HI, O'Brien WJ (1992a) Foraging and search behaviour of golden shiner (Notemigonus crysoleucas) larvae. Can J Fish Aquat Sci 49:813-819

Browman HI, O'Brien WJ (1992b) The ontogeny of search behaviour in the white crappie, Pomoxis annularis. Envir. Biol Fish 34:181-195

Coughlin DJ (1993) Prey location by clownfish (Amphiprion perideraion) larvae feeding on rotifers (Brachionlis plicatilis). J Plankton Res 15:117-123

Dower J, Miller TJ, Leggett WC (in press) The role of microscale turbulence in the feeding ecology of larval fish. Adv Mar Biol

Hwang JS, Costello JH, Strickler JR (1994) Copepod grazing in turbulent flow: elevated foraging behaviour and habituation of escape responses. J Plankton Res 16:4221-4231

Jenkinson IR (1995) A review of two recent predation-rate models: the dome-shaped relationship between feeding rate and shear rate appears universal. ICES J Mar Sci 52 $605-610$

Kiorboe T, Mackenzie BR (1995) Turbulence-enhanced prey encounter rates in larval fish: effects of spatial scale, larval behaviour and size. J Plankton Res 17:2319-2331

Landry F, Miller TJ, Leggett WC (1995) The effects of smallscale turbulence on the ingestion rate of fathead minnow (Pimephales promelas). Can J Fish Aquat Sci 52 $714-1719$

MacKenzie BR, Kiorboe T (1995) Encounter rates and swimming behaviour of pause-travel and 'cruise' larval fish predators in calm and turbulent environments. Limnol Oceanogr 40:1278-1289

Mackenzie BR, Miller TJ, Cyr S, Leggett WC (1994) Evidence for a dome-shaped relationship between turbulence and larval fish ingestion rates. Limnol Oceanogr 39:1790-1799

Marrasé C, Costello JH, Granata T, Strickler JR (1990) Grazing in a turbulent environment: II. Energy dissipation, encounter rates and the efficacy of feeding currents in Centropages hamatus. Proc Natl Acad Sci USA 87 $1653-1657$

Muelbert JH, Lewis MR. Kelley DE (1994) The importance of small-scale turbulence in the feeding of herring larvae. $\because$ Plankton Res 16:927-944

O'B:ien WJ, Browman HI, Evans BI (1990) Search strategies of foraging animals. Am Sci 78:152-160

Rosenthal H. Hempel G (1970) Experimental studies in feeding and food requirements of herring larvae (Clupea harengus L.). In: Steele JH (ed) Marine food chains. Oliver \& Boyd, Edinburgh, p 344-364

Saiz E (1994) Observations of the free-swimming behaviour of Acartia tonsa: effects of food concentration and turbulent water motion. Limnol Oceanogr 39:1566-1578

Sundby $\mathrm{S}$ (1995) Wind climate and foraging of larval and juvenule Arcto-Norwegian cod (Gadus morhua L.). Can J Fish Aquat Sci 121:405-415

Sundby S, Ellertsen B, Fossum P (1994) Encounter rates between tirst-feeding cod larvae and their prey during moderate to strong turbulent mixing. ICES Mar Sci Symp $198: 393-405$ 